$$
\text { DOE/ER/60991- - T1 }
$$

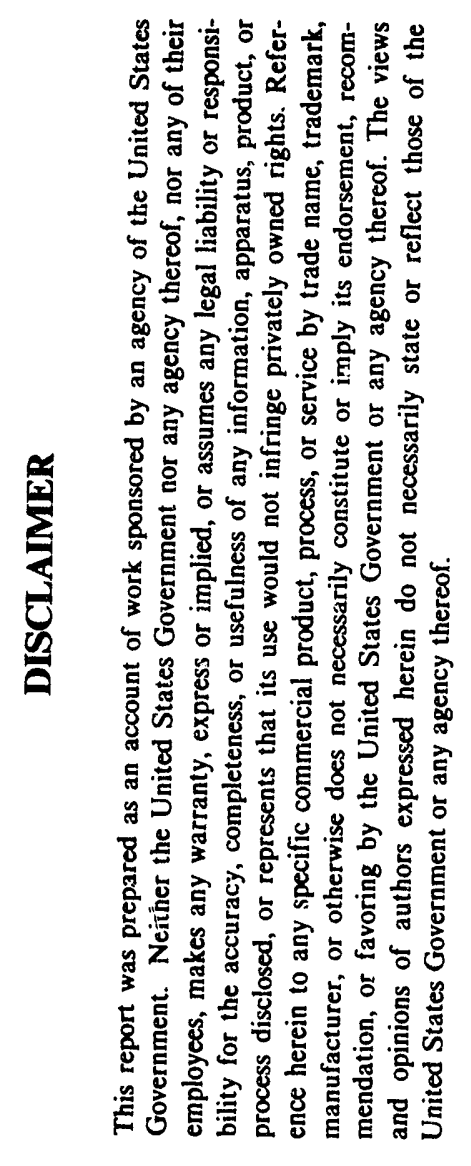

\section{PHYSIOLOGICALLY ANAEROBIC MICROORGANISMS OF THE DEEP SUBSURFACE}

\author{
Progress Report \\ June 1, 1990 - May 30, 1991
}

S. E. Stevens, Jr. and K.-T. Chung Department of Biology Memphis State University Memphis, Tennessee 38152

June 1991
PREPARED FOR THE U.S. DEPARTMENT OF ENERGY UNDER GRANT NUMBER DE-FG05-90ER60991

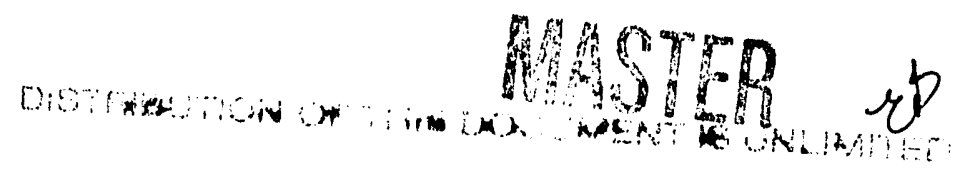


Herein, I summarize the progress made by the research team assembled at Memphis State University during the first 10 months of our ongoing DOE sponsored project titled "Physiologically Anaerobic Microorganisms of the Deep Subsurface" (DOE Contract No. DE-FG05-90ER60991) .

SCHEDULE OF MAJOR RESEARCH ACTIVITIES

Year $1(1990-91)$

- Determine numbers, diversity, and morphology of anaerobic microorganisms in 15 samples of subsurface material from the Idaho National Engineering Laboratory (INEL), in 18 samples from the Hanford Reservation (HR) and in 1 rock sample from the Nevada Test Site (NTS); set up long term experiments on the chemical activities of anaerobic microorganisms based on these same samples; work to improve methods for the micro-scale determination of in situ anaerobic microbial activity; begin to isolate anaerobes from these samples into axenic culture; and, begin to identify the axenic isolates.

Year $2(1991-92)$

- Determine selected physiological characteristics of all isolates from INEL, HR, and NTS; analyze the chemical activities of anaerobic microorganisms from the long term experiments; continue development of micro-scale methods; compare isolates from different depths at each site; publish fundamental results from the above work.

Year $3(1992-93)$

- Look for possible new anaerobic isolates from long term 
enrichments in stored samples; characterize these as above, if found; begin studies leading to the long term preservation of each axenic culture; analyze data sets from each investigator and determine factors which influence community structure especially in regard to anaerobic microorganisms; transfer preserved isolates to the culture collection at Florida State University; publish additional results.

\section{PROJECT OUTPUT: MAJOR ACCOMPLISHMENTS $(6 / 90-3 / 91)$}

- We have completed plate counts, MPN's, spin-tube counts, acridine orange direct counts, and ATP estimates on 15 samples from the INEL site, 18 samples from the HR site, and 1 sample from the NTS site.

A flow chart summarizing sample manipulation is shown in Fig. 1. Medium 1 is an egg yolk emulsion medium recommended as a general medium for anaerobic bacteria. Medium 2 is a 1:50 dilution of medium 1. Medium 3 is full strength PTYG. Medium 4 is a 1:100 dilution of medium 3. Both spread plates and spin-tubes with pre-reduced (bubbled with $4 \% \mathrm{H}_{2}$ balance $\mathrm{CO}_{2}$ ) medium were used for counting purposes. The use of PTYG as a growth medium for anaerobic bacteria was validated by plating pure cultures of anaerobic bacteria obtained from the ATCC on both their ATCC recommended medium and on PTYG. The results support the use of PTYG as a plating medium for anaerobes (see Tables 1-3). In addition, we used Postgate's medium to determine the presence of 


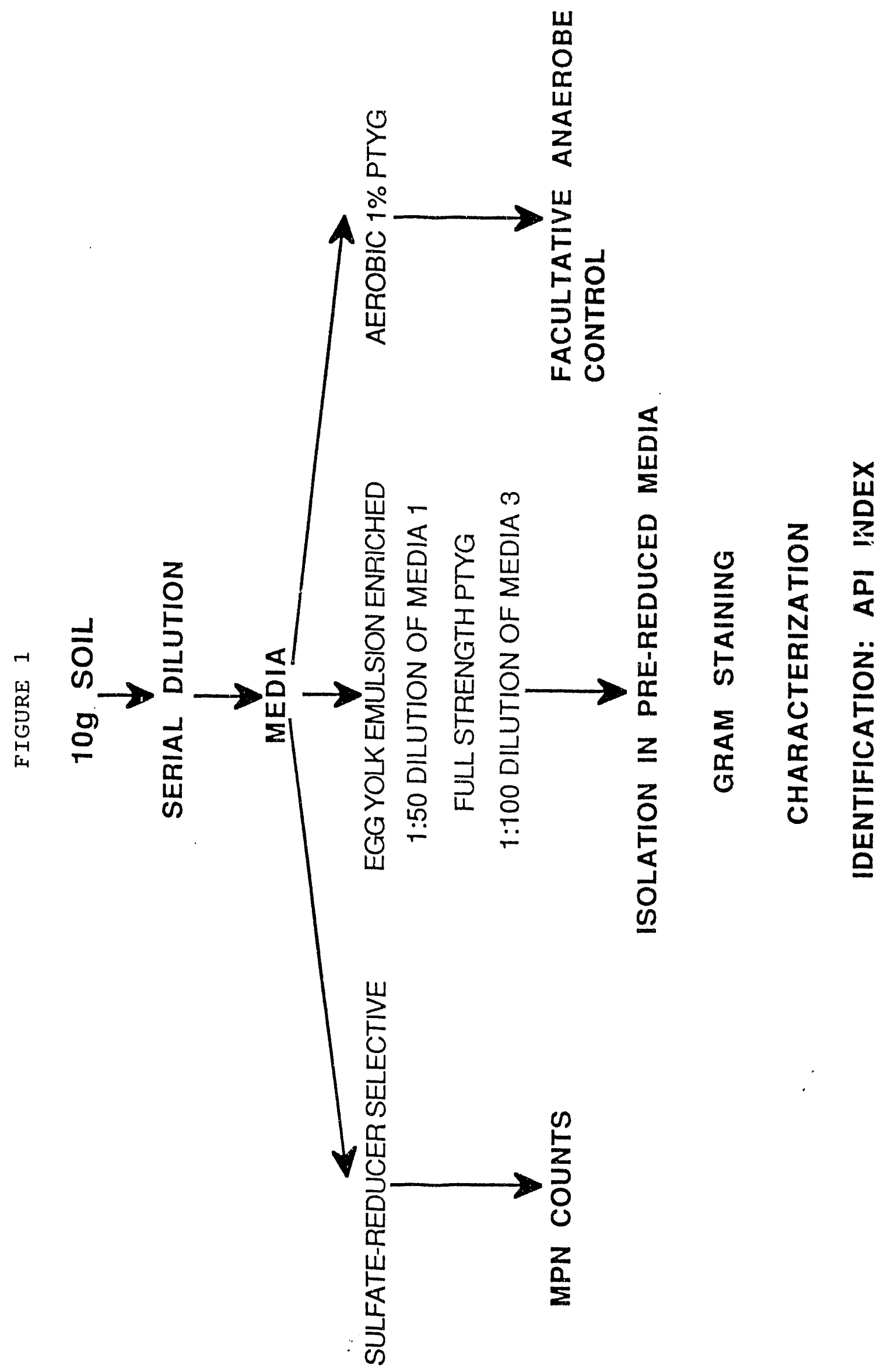




\begin{tabular}{|l|c|}
\hline \multicolumn{1}{|c|}{ MEDIUM } & CULTURE COUNT (PER ML) \\
\hline REINFORCED & $9.5 \times 10^{8}$ \\
CLOSTRIDIAL MEDIUM & $6.8 \times 10^{8}$ \\
(OXOID) & $1.3 \times 10^{9}$ \\
\hline TPYG & $1.2 \times 10^{9}$ \\
& $1.1 \times 10^{9}$ \\
& $1.1 \times 10^{9}$ \\
\hline NUTIENT AGAR & $<10^{5}$ \\
\hline LACTOBACILLUS AGAR & $1.1 \times 10^{9}$ \\
& $7.8 \times 10^{8}$ \\
& $7.7 \times 10^{8}$ \\
\hline MEDIUM 1 & NOGROTH \\
\hline
\end{tabular}

\section{BACTEROIDES FRAGILIS ATCC 23745 GROWN ON BHI FOR 48 HOURS.}




\begin{tabular}{|l|c|}
\hline MEDIUM & CULTURE COUNT (PER ML) \\
\hline REINFORCED & $1.1 \times 10^{7}$ \\
CLOSTRIDIAL MEDIUM & $1.1 \times 10^{7}$ \\
(OXOID) & $2.9 \times 10^{7}$ \\
\hline TPYG & $1.1 \times 10^{7}$ \\
& $8.8 \times 10^{6}$ \\
& $7.5 \times 10^{6}$ \\
\hline NUTRIENT AGAR & $<10^{5}$ \\
\hline LACTOBACILLUS & $8.0 \times 10^{5}$ \\
AGAR & $4.5 \times 10^{5}$ \\
& $3.5 \times 10^{5}$ \\
\hline
\end{tabular}

\section{CLOSTRIDIUM PERFRINGEN ATCC 3624 GROWN ON BHI FOR 24 HOURS.}


TABLE 3

\begin{tabular}{|l|l|}
\hline MEDIUM & \multicolumn{2}{|c|}{ CULTURE COUNT (PER ML) } \\
\hline LACTOBACILLUS AGAR $^{*}$ & $1.5 \times 10^{5}$ \\
& $3.2 \times 10^{6}$ \\
& $4.7 \times 10^{6}$ \\
\hline TYPG* $^{*}$ & $5.5 \times 10^{4}$ \\
& $6.0 \times 10^{4}$ \\
& $6.0 \times 10^{4}$ \\
\hline \multirow{2}{*}{ MEDIUM I** $^{*}$} & $4.4 \times 10^{5}$ \\
& $9.9 \times 10^{5}$ \\
& $7.9 \times 10^{5}$ \\
\hline MEDIUM II** & $2.7 \times 10^{5}$ \\
& $6.5 \times 10^{5}$ \\
\hline
\end{tabular}

\section{LACTOBACILLUS BULGARICUS ATCC 27558 GROWN ON BHI FOR 24 HOURS}


sulfate reducing bacteria. To date we have found none. We also used 2 media, $\mathrm{CO}_{2} / \mathrm{H}_{2}$ and acetate/methylamine to determine the presence of methanogens and acetogens in INEL, HR, and NTS samples.

Plate count data, MPN data, spin-tube counts and AODC's for 15 samples from INEL and 18 samples from HR are given in Tables 4 and 5. ATP estimates for these same samples are not given because none of them, with the exception of the spiked sample from INEL, indicated a reliably significant concentration of ATP.

The NTS sample provided by Dr. Penny Amy has not shown any anaerobic growth as yet.

Methane production occurred in two HR samples and gas was taken up in an additional HR sample. These results indicate methanogenic bacteria is samples $0007 \mathrm{~b}$ and 0307 and a probable acetogenic bacterium in sample 0407 from HR. AODC data are plotted in Figs. 2 and 3 as number of cells versus depth. The highest numbers of cells were between 200 and 300 feet below the land surface at both INEL and HR.

- We have recorded the colonial morphology, cellular morphology, and Gram reaction on each colony type observed on plates and/or spin-tubes. These results also give us a preliminary notion of diversity.

Pie charts summarize our results and are presented in Fig. 4. A bit better than $30 \%$ of all isolates to date from both the INEL and HR sices were Gram (-), long, unicellular rods. The remaining cellular types ( $70 \%$ of the isolates) 
选 $m$

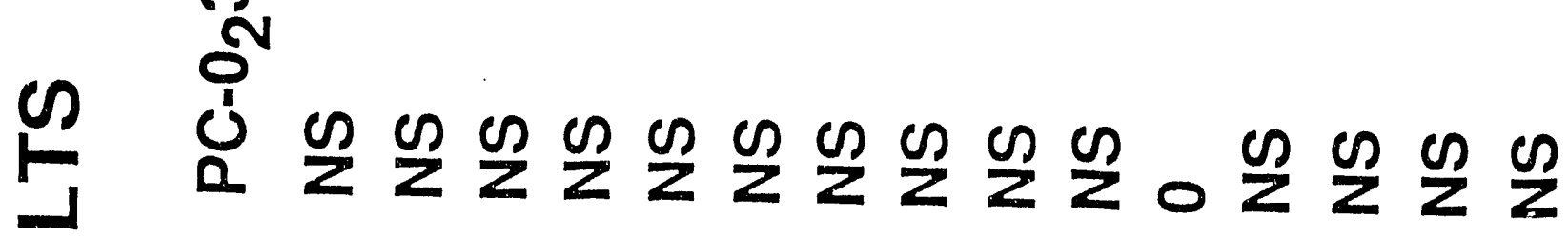

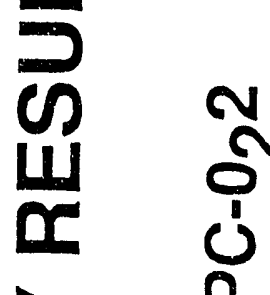

$>$

ac

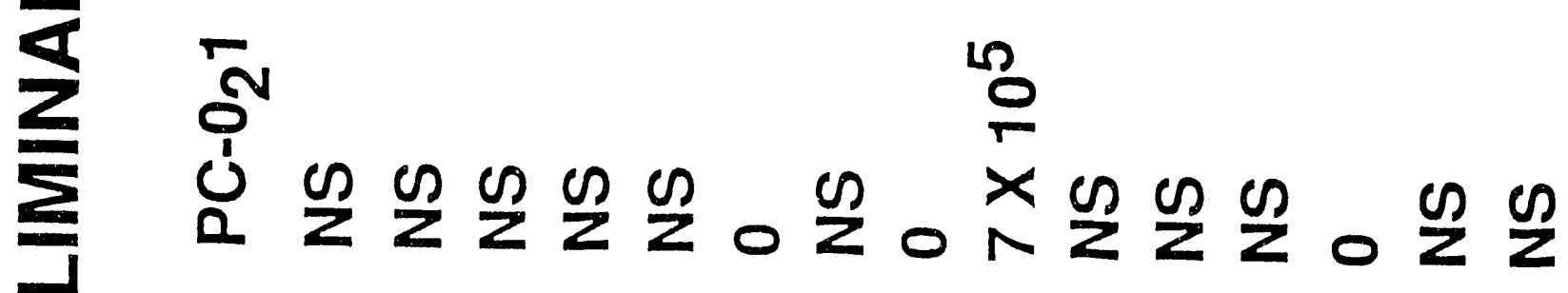

ш

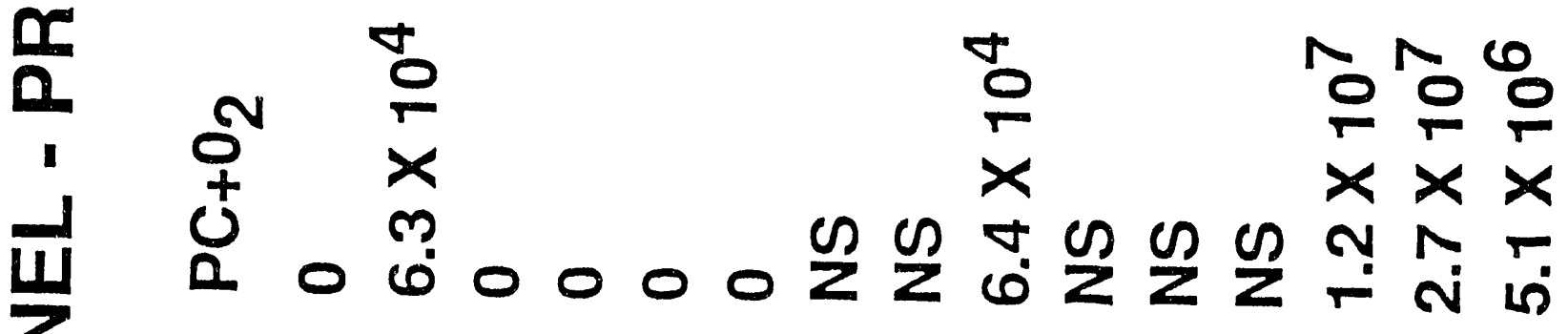

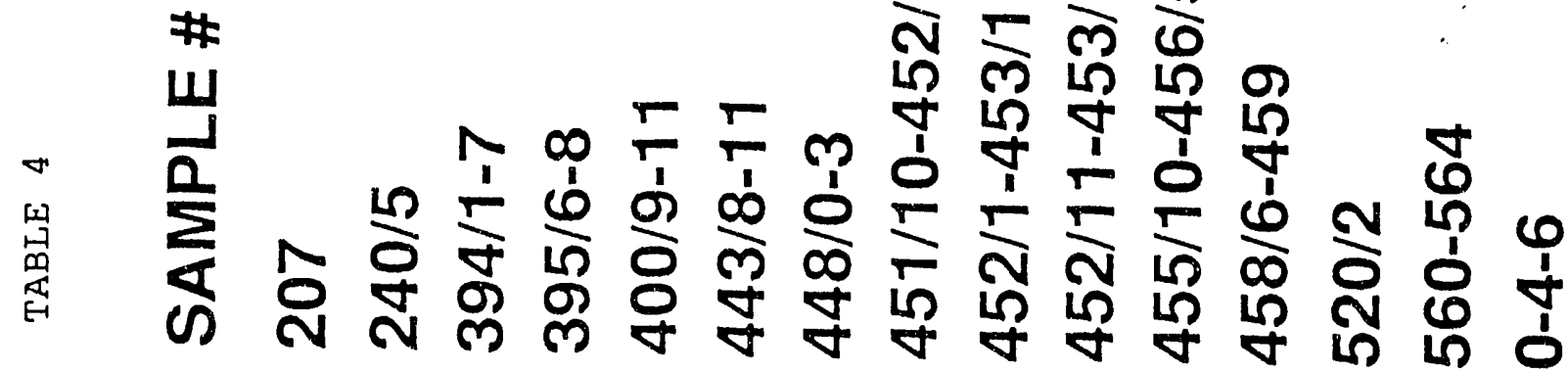


$\operatorname{li}_{0}^{\infty} \hat{O}^{N}$

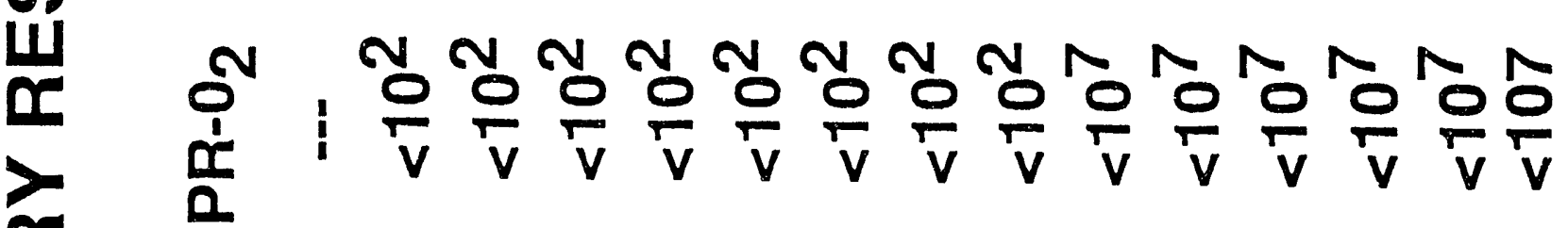

$\sum \frac{0}{11}$

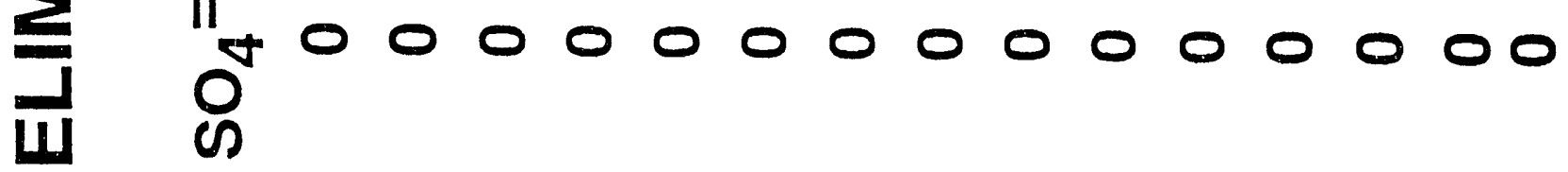

ac

a.

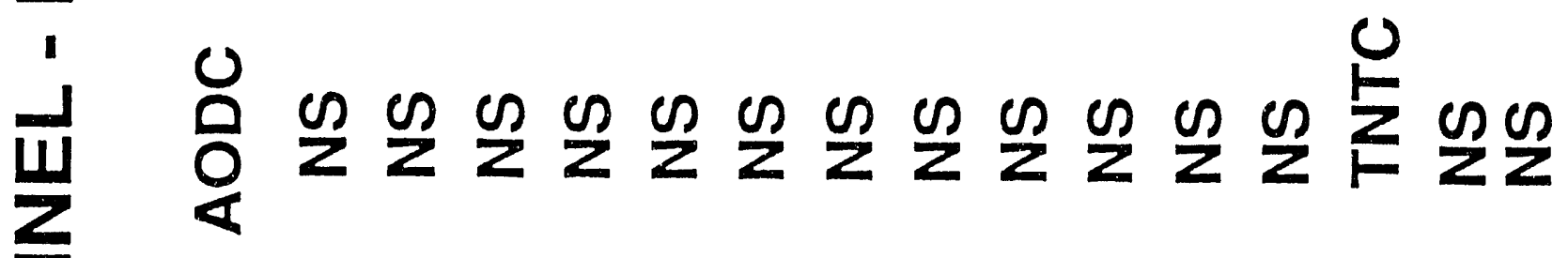

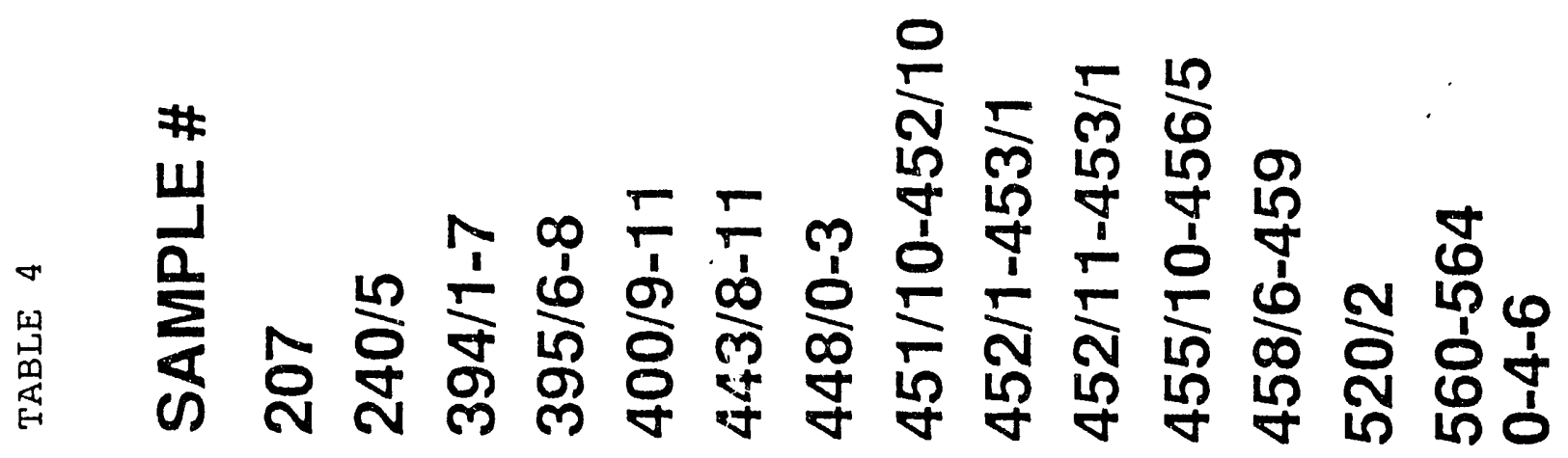




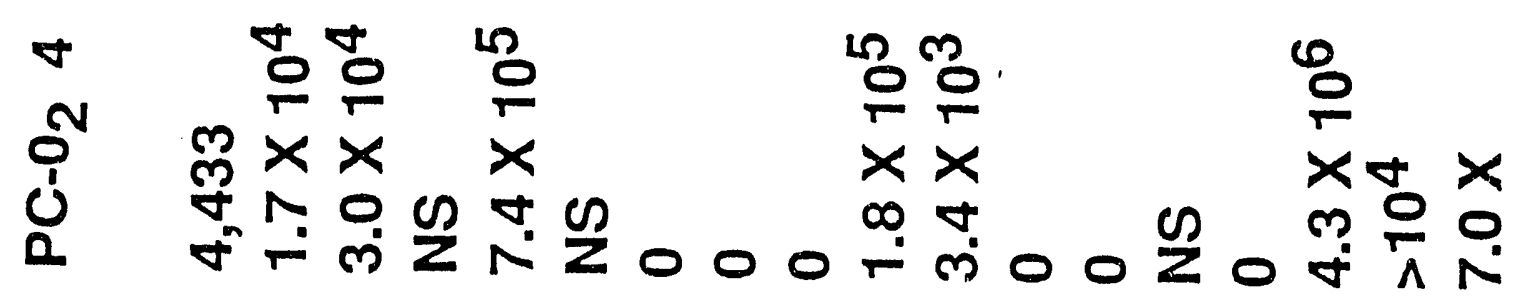

兽

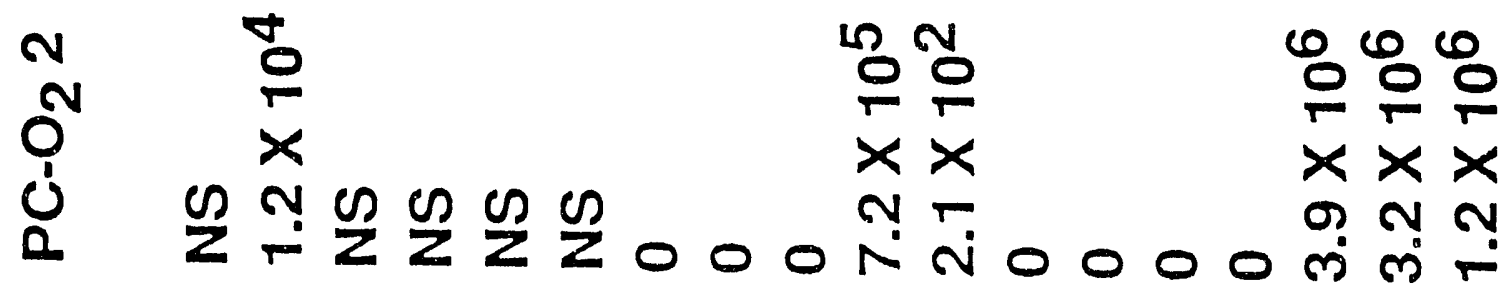

总

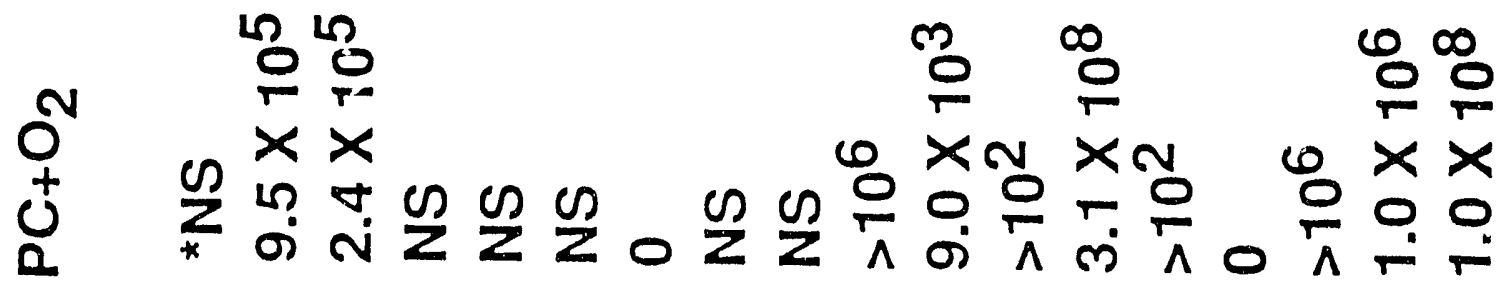

\#

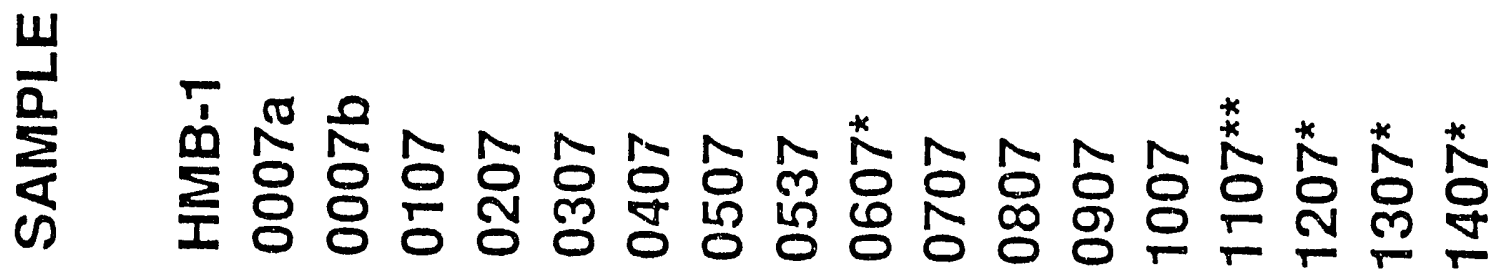




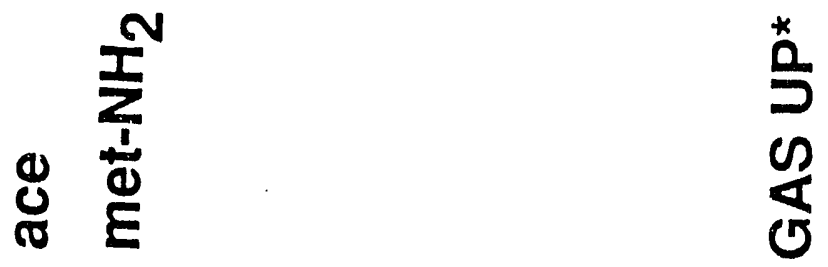

$\overbrace{0}^{\mathbf{N}}$

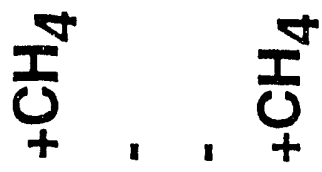

$\frac{0}{a}$

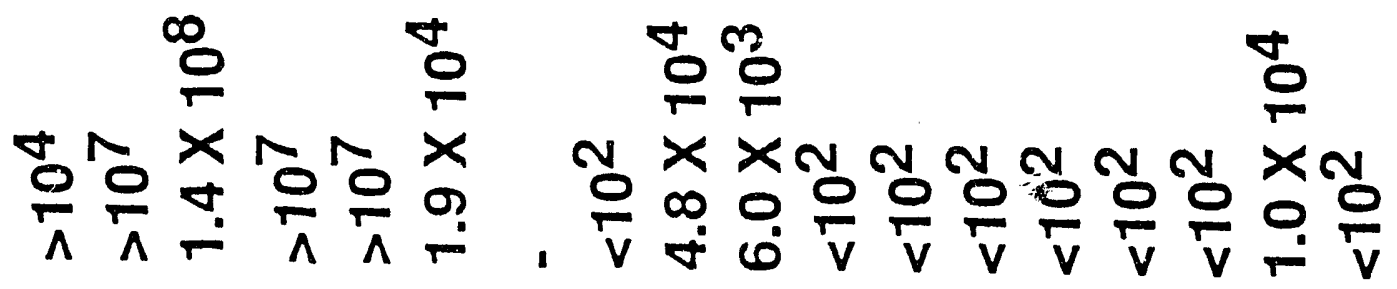

${ }_{0}^{11}$

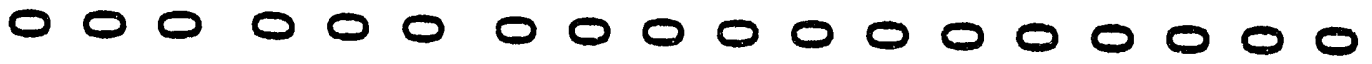

$\frac{0}{0}$

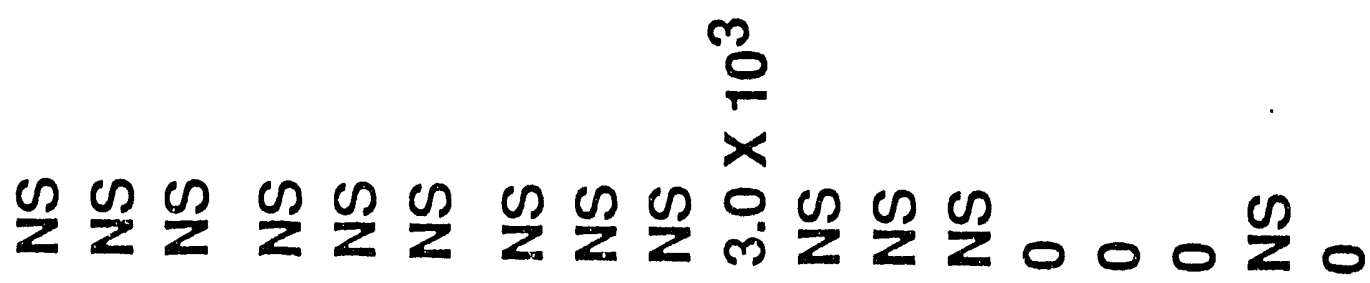

\#

崖

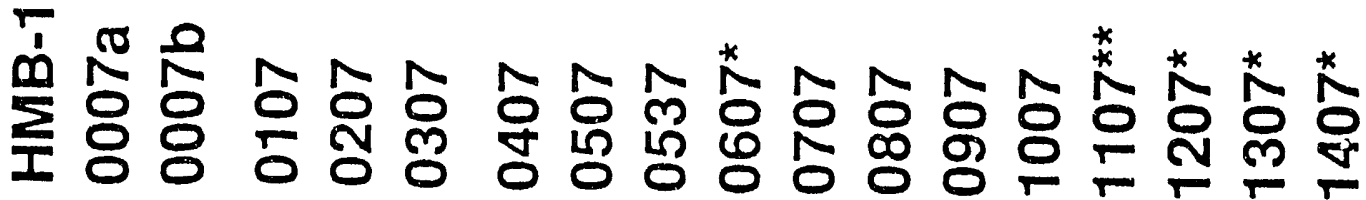




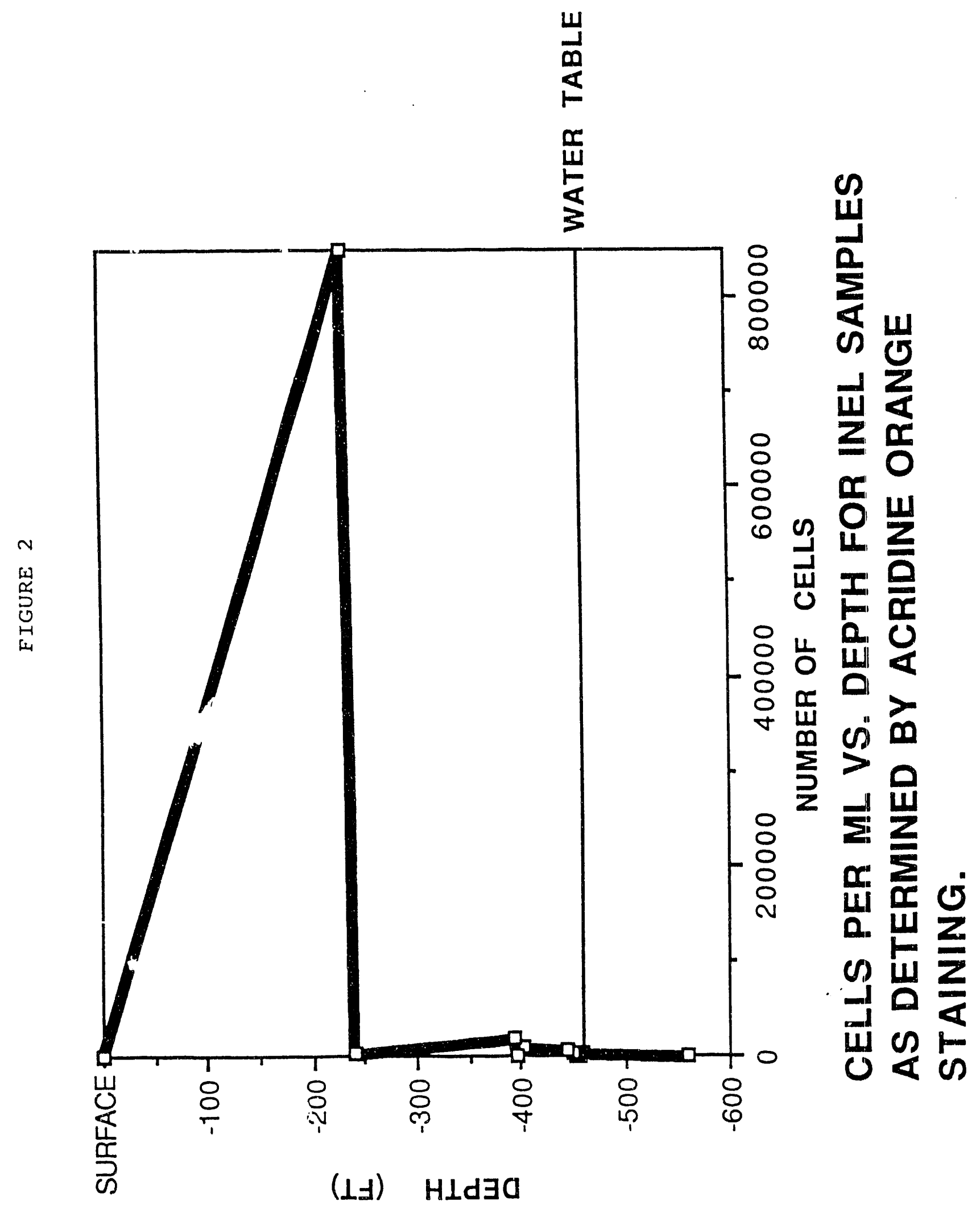




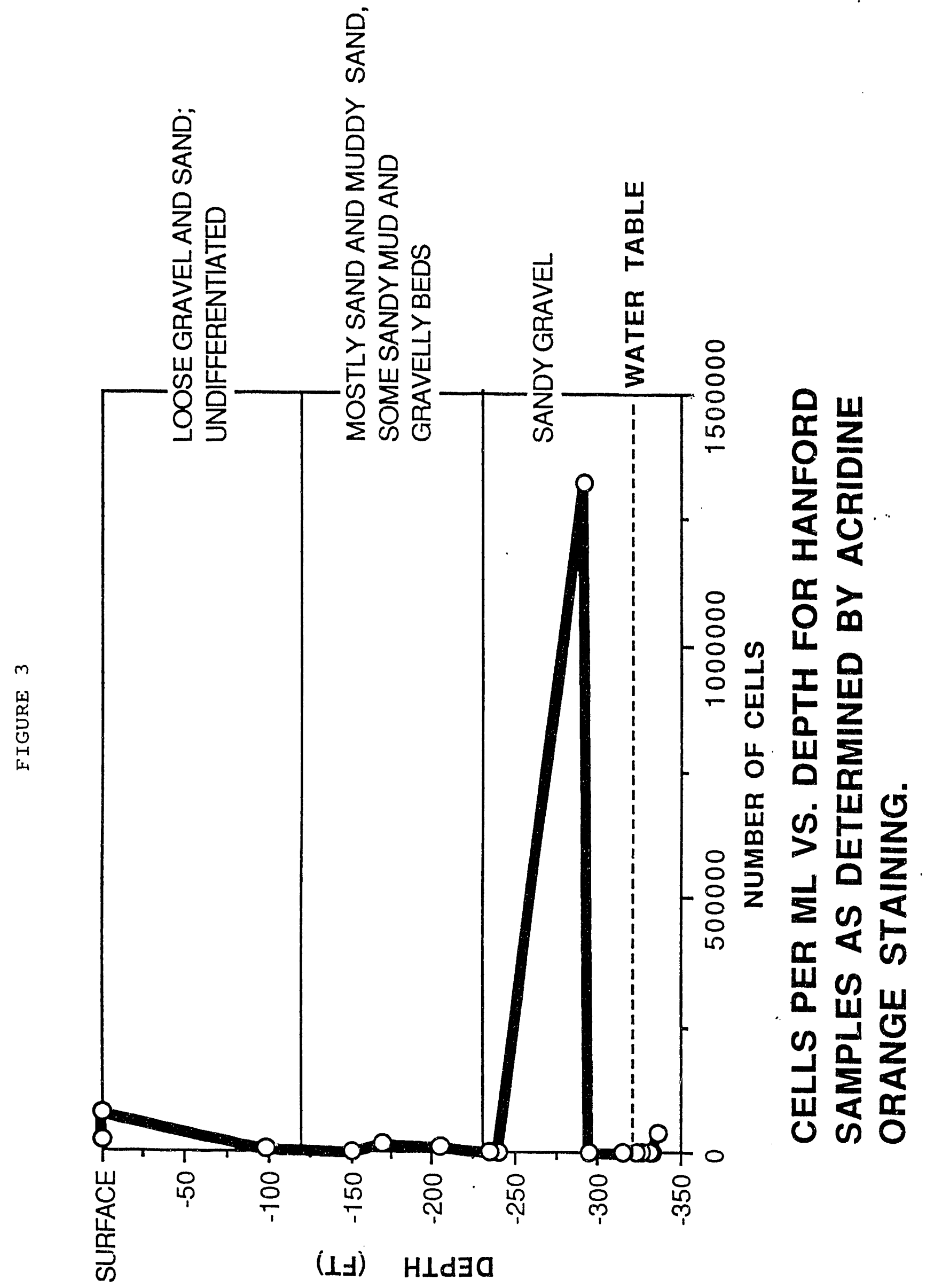




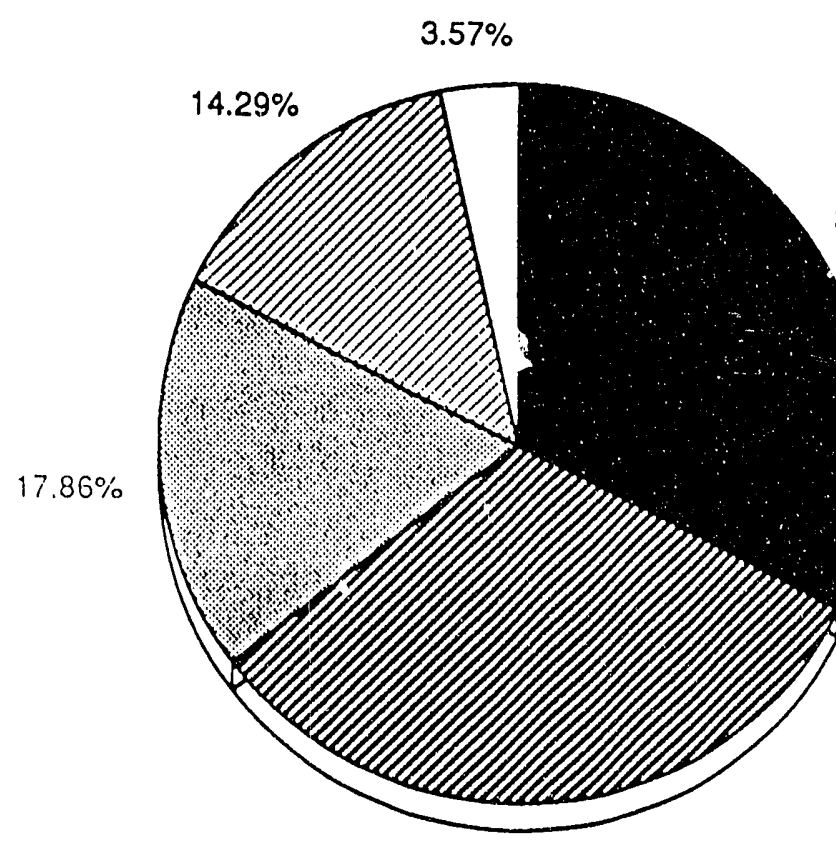

$32.14 \%$

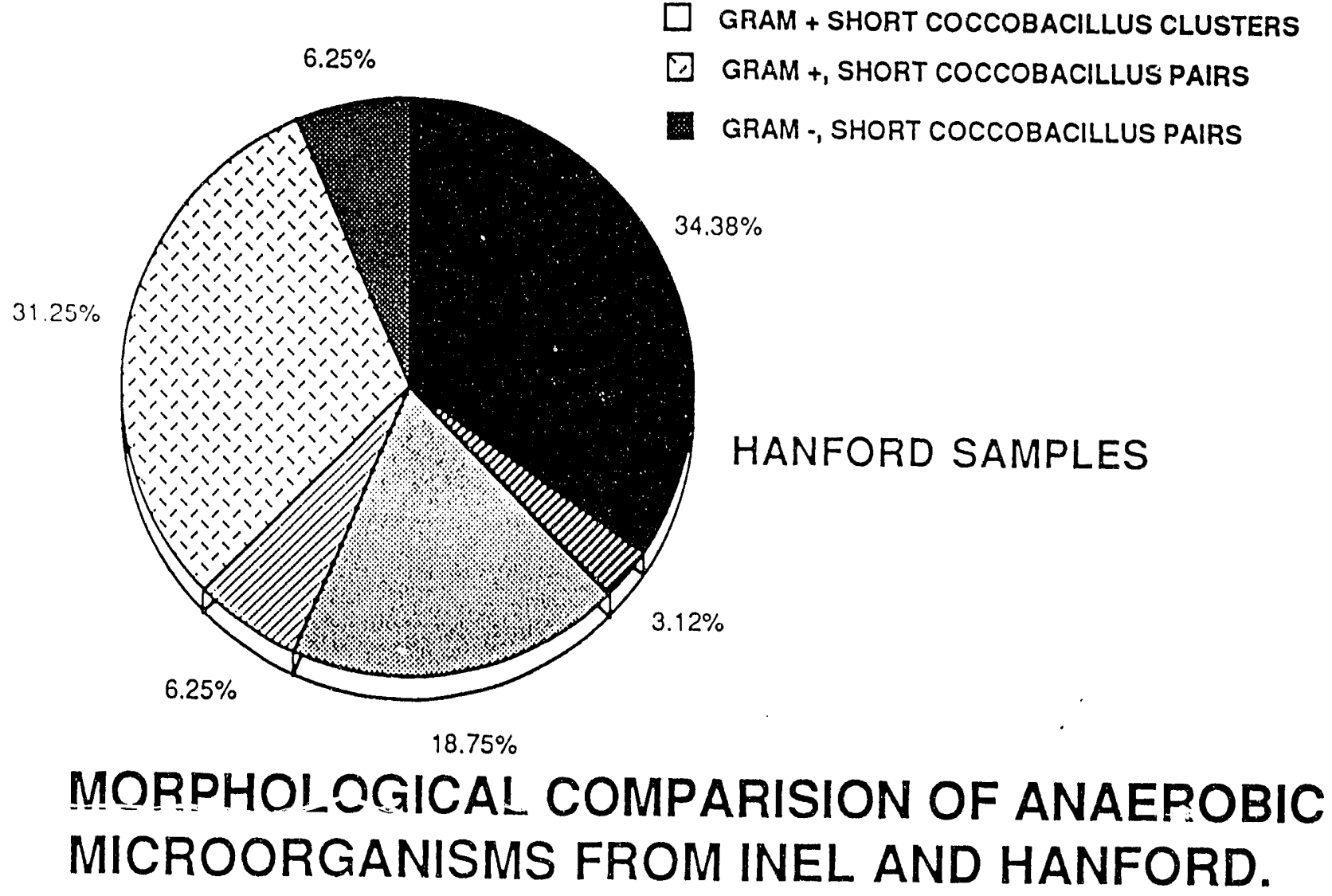

INEL SAMPLES

GRAM - LONG SINGLE RODS

GRAM + LONG SINGLE RODS

GRAM + SHORT SINGLE COCCOBACILLUS

GRAM + SHORT COCCOBACILLUS CLUSTERS

GRAM +, SHORT COCCOBACILLUS PAIRS

GRAM -, SHORT COCCOBACILLUS PAIRS
G GRAM + SHORT COCCOBACILLUS CHAINS

HANFORD SAMPLES 
were strikingly different between the two sites.

- So far, we have isolated 28 apparently distinct anaerobes from all the samples examined. In addition, we have evidence for methanogens and an acetogen although we do not yet have axenic cultures of these.

- We have begun to obtain physiological/biochemical profiles of our axenic cultures.

- The data we have has been coded and entered into dBASE III Plus.

- We have initiated microelectrode experiments of selected samples to see if microbial activity is detectable with time. No results are available.

- Presentations on this work:

(i) Poster presentation by E.B. Sullivan, T.T. Hendrix, K. -T. Chung, and S.E. Stevens, "Isolation and characterization of anaerobic microorganisms from the deep subsurface." Third Annual Graduate Research Forum, Memphis state University, March 26, 1991.

(ii) Poster presentation as above at the annual meeting of the Western Collegiate Division of the Tennessee Academy of Science, Rhodes College, April 13, 1991. 

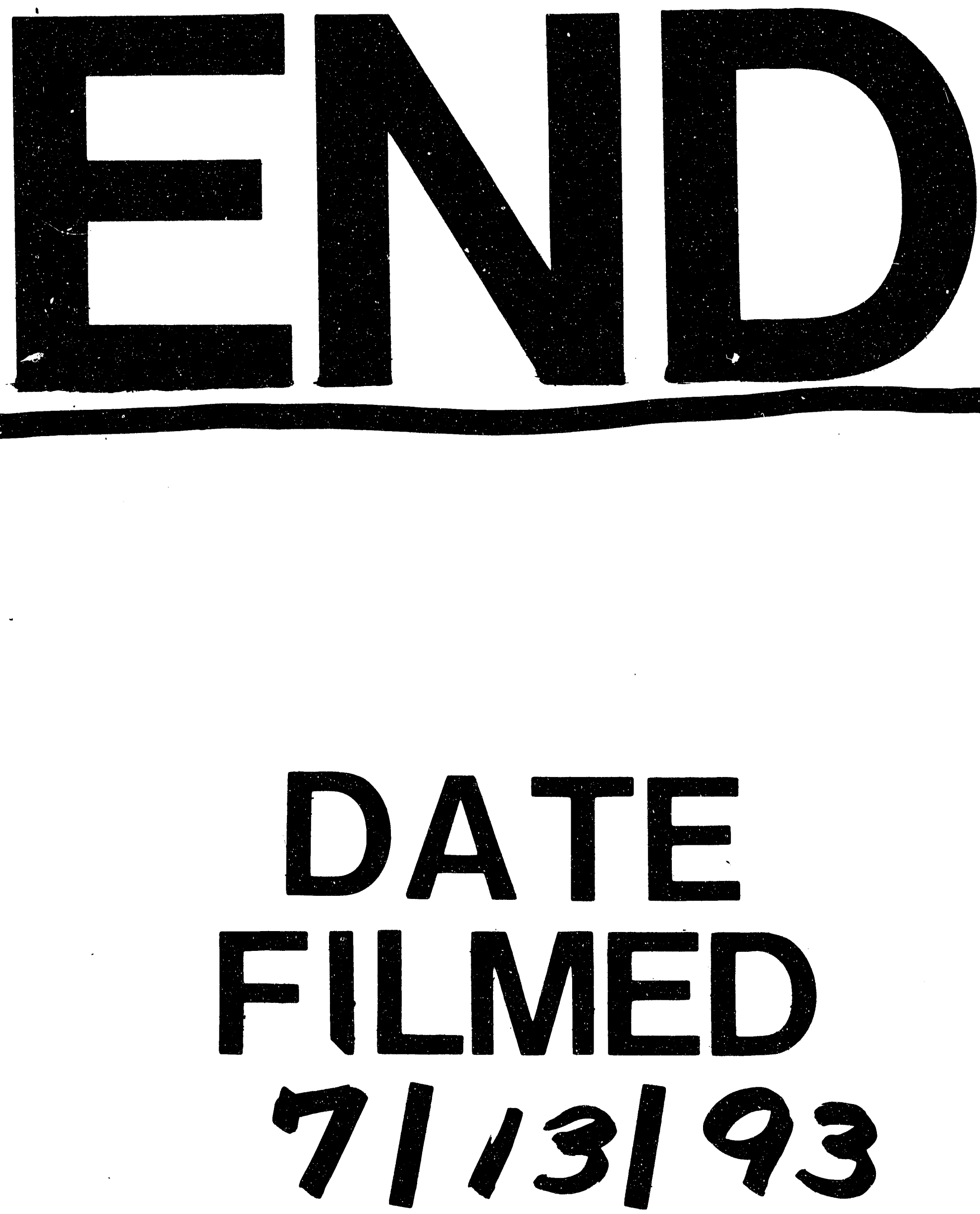
-

. 